\title{
Developing Retina and PNS Segments for Transplantation Into the Adult Host Eye: Reconstruction of the Mammalian Visual System. 1. Methodology
}

\author{
Michael F. Zanakis, Ph.D.*, Howard F. Lowe, Glenn Jacobsen, Michael LaCorte, \\ Simone P. Lee and Brian H. Hallas, Ph.D. \\ *Department of Bioengineering, School of Engineering, The Cooper Union, \\ 51 Astor Place, New York, NY 10003 and Department of Anatomy and Neuroscience, \\ New York College of Osteopathic Medicine, Old Westbury, NY 11756, U.S.A.
}

\begin{abstract}
Various techniques have been explored to determine the uses and limitations of techniques that enable the adult CNS to regenerate, but relatively little attention has been given to the consideration of a "reconstructed" visual system. Using this approach, one can design experiments to study the uses of exogenous tissues in reestablishing neuronal circuits that have been damaged. Toward this end, experiments were designed to determine whether embryonic retinal ganglion cells can project axons into a grafted PNS "bridge", and enter adult host targets that were partially deafferented. Embryonic eyes of E11, E14, E18 and E21 rats were sutured to peripheral nerve segments which served as bridges between the host eye and frontal cortex. Projections between the developing retina and the host brain could then be evaluated using HRP tracing techniques. From a methodological standpoint, the preparations are $65 \%$ effective; i.e., a viable
\end{abstract}

Reprint address:

Michael F. Zanakis, Ph.D.

Department of Neuroscience

New York College of Osteopathic Medicine

Old Westbury, NY 11756

U.S.A. bridge results between the embryonic eye and the host forebrain. The results presented in the accompanying paper demonstrate that the technique can yield results indicative of embryonic retinal development and axonal projection through the graft and into the host brain. This partial reconstruction of the visual system may prove a useful tool in understanding the uses and limitations of grafting in the CNS.

\section{KEY WORDS:}

Embryonic, Transplantation, Retina, Regeneration

\section{INTRODUCTION}

In lower vertebrates, axotomized neurons have the capability to regenerate and form functional contact with the previously denervated target organ. In these animals, the peripheral nervous system (PNS) and the central nervous system (CNS) are both quite capable of this. In mammals, the PNS does retain the ability to regenerate following axotomy of neurons. Unfortunately, the adult mammalian CNS cannot easily accomplish this task.

Generally speaking, any attempt of adult mammalian CNS neurons to regenerate dam- 
aged axons is often aborted. Whether the inability of the CNS to regenerate like the PNS is related to adverse micro-environmental conditions or to an inherent inability of most CNS neurons to regrow is still unknown. However, attempts to understand the cellular events in CNS injury and regeneration have been rapidly expanding.

In order to gain insight into the mechanisms driving the regenerative phenomenon (and the reasons for its difficulty), various models of CNS injury have been used. The results from a large number of investigators have shown that, unlike the previous assumptions of past decades, certain portions of the CNS are indeed capable of limited regeneration under certain conditions. One model of CNS injury centers around the use of the mammalian optic nerve, which provides easy surgical access and a relatively simple structure, thereby facilitating manipulation and study.

Recent studies have attempted to induce regeneration by transplanting embryonic tissue in the damaged region to act as bridges or scaffolds for regenerating neurites /9/. Transplantation of embryonic tissues has proven to be a useful tool for determining the essential factors for nervous system reconstruction (see $/ 5 /$ for review). Since embryonic cells have a great potential for growth and differentiation, their transplantation appears to provide a suitable environment capable of promoting and supporting growth of the lesioned adult central nervous system $/ 6,7 /$.

The anterior chamber of the rodent eye is quite capable of accommodating a small piece of transplanted tissue. Indeed, various tissue types have been transplanted into the anterior chamber by several investigators $/ 6-8,14 /$. Of the tissues studied, relatively little attention has been given to the in oculo transplantation of retina.

The following report describes the use of a novel technique to bridge the embryonic and adult CNS using a PNS scaffold. The technique allows one to determine the growth potential of various age embryonic retinae when transplanted into the anterior eye chamber and sutured to a peripheral nerve bridge.

\section{MATERIALS AND METHODS}

The following procedure involves four steps. Please refer to Figure 1 for schematic representation.

\section{Removal of Embryonic Eyes}

Under ketamine anesthesia $(100 \mathrm{mg} / 100 \mathrm{~g}$; Ketalar, Parke Davis), supplemented with Rompun

1

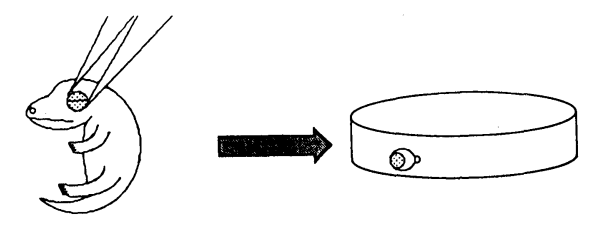

2

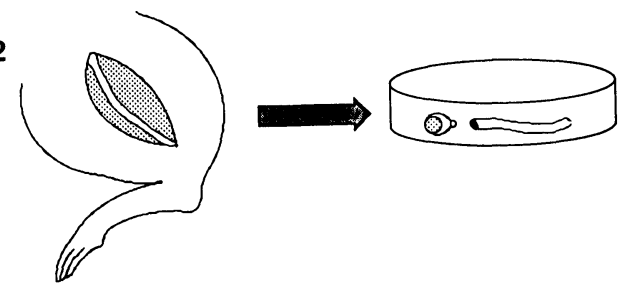

3

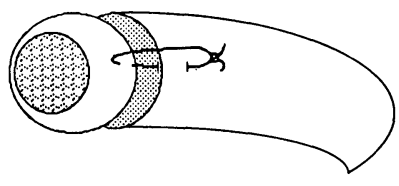

4

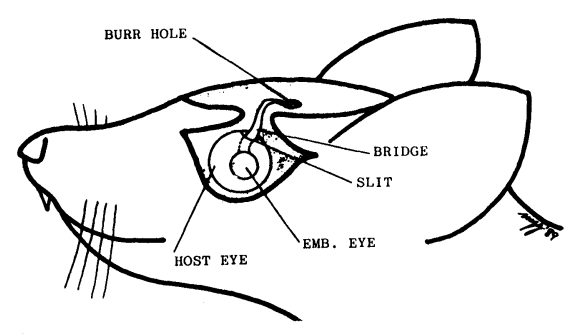

Fig. 1: Schematic diagram depicting sequence of events necessary for the procedure; 1 ) embryonic eye is removed and placed in a culture dish containing lactated Ringer's solution, 2) tibial nerve is removed and placed in the culture dish containing the embryonic eye 3 ) embryonic eye is sutured to the distal end of the tibial nerve, 4) embryonic eye/nerve complex is inserted into the host eye while the opposite end of the nerve is inserted into a burr hole over the frontal cortex. 
(30mg/100g; Haver-Lockhart) the abdomen of pregnant female Wistar rats (approximately $300 \mathrm{~g}$ ) were opened to remove embryos of age E11, E14, E18 and E21. The eyes of the embryos were removed by cutting the overlying skin of the eye, and carefully dissecting around and under the globe with iridectomy scissors. Removal of the eye can be performed with 3-4 cuts of the scissors. These embryonic eyes were then placed into a culture dish containing lactated Ringer's solution at room temperature, where the globe was cleared of loosely attached tissue. Clearing of the tissue is most easily performed when the globe is submerged in the Ringer's solution. All work in vitro is best performed on a black surface, which provides contrast between the tissue and the surrounding medium.

\section{Removal of Tibial Nerves for Bridges}

Adult male Wistar rats $(250-300 \mathrm{~g})$ were anesthetized as above. The sciatic nerves were exposed at mid thigh, and a $4.0 \mathrm{~cm}$ long segment was removed. This segment was then placed into a culture dish containing Ringer's solution. The tibial nerve was isolated from the other nerves by carefully cutting away the other fascicles with a No.11 scalpel. The entire procedure is best performed while submerged in Ringer's. The tibial nerve is preferable since it is generally larger than the peroneal nerve. The epineurium was then stripped from the tibial nerve by "skinning", i.e., where the nerve is held proximally with fine forceps while the epineurium was pulled from the proximal end. This leaves the nerve with only the perineurium as the outer coat. The end of the nerve that was held (resulting in being crushed or mashed) was cut off, leaving a cleanly cut stump at either end. The nerve segment that results after skinning and isolation is approximately $3.5 \mathrm{~cm}$ long.

\section{Preparation of Transplant}

All removed tissue was placed into a single culture dish containing Ringer's solution. In this dish, and with the aid of a dissecting microscope
(Ausjena) and jeweler's forceps, the embryonic eye was sutured to the distal end of the tibial nerve. Distal orientation is important since growing axons would enter one side of the nerve and would not get redirected into another fascicular branch (i.e., they will emerge somewhere from the large tibial stump) as may occur in the reverse orientation. Suturing was accomplished by first aligning the eye so that the optic nerve was near the tibial nerve stump. Then, a fine suture needle with attached 8-0 silk thread (G7, Ethicon) was passed through one side of the scleral portion of the eye, and then through the perineurium at the distal stump end of the tibial nerve segment. By gently pulling the suture, the eye closely apposed the nerve and a single tie held the two together. A similar suture firmly attaches the opposite side of the eye to the nerve stump (see Figure 2) so that the optic nerve is abutted against the stump of the tibial nerve. As

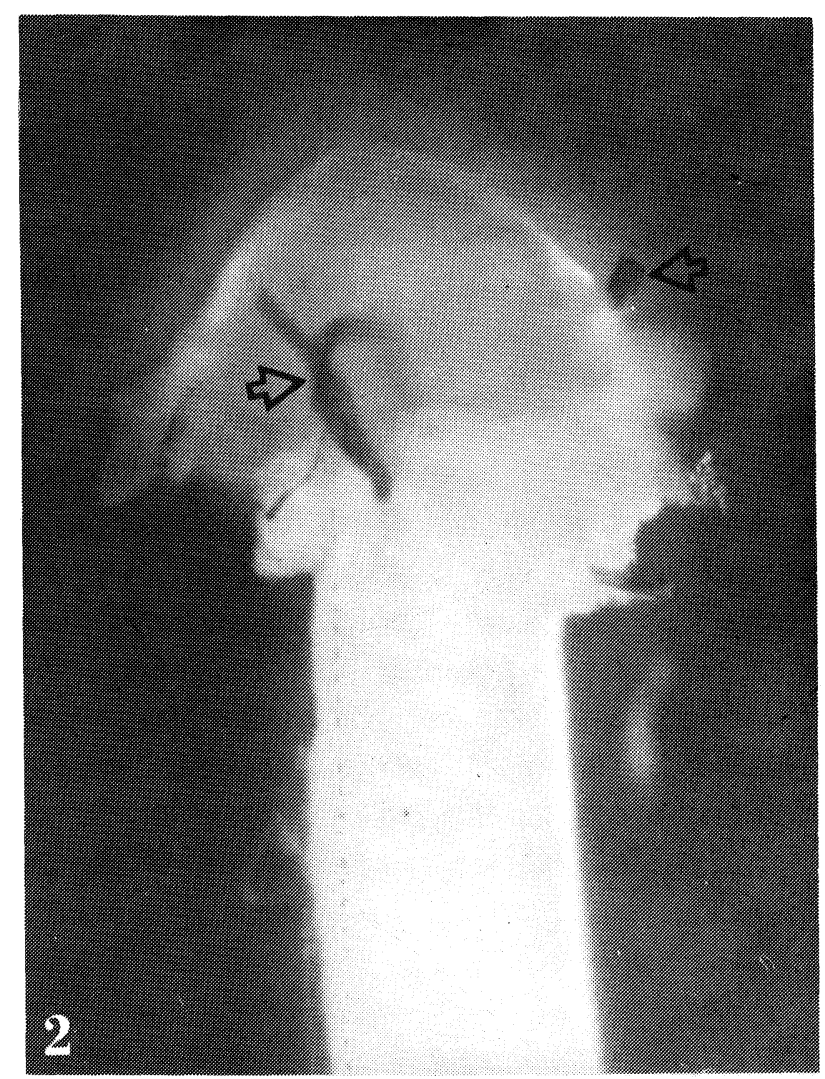

Fig. 2: Low power photomicrograph of an E11 eye sutured to the distal end of the tibial nerve. Diameter of nerve is approximately $1 \mathrm{~mm}$. Arrows denote sutures. 
complement of the optic nerve on the central side of the transection, and significantly degenerates the axons and retinal ganglion cells of the host.

All animals were allowed to recover from anesthesia on a heating pad set at $37^{\circ} \mathrm{C}$. Thereafter, all animals were maintained in a $22-25^{\circ} \mathrm{C}$ natural light cycle room, and fed Lab Chow ${ }^{\mathrm{TM}}$ (Purine) and water ad libitum. The animals were housed two per cage, since this limits mortality. After 30,90 or 180 days, euthanasia was performed by sodium pentobarbitol (Nembutal) overdose, which is consistent with the recommendations of the Panel on Euthanasia of the American Veterinary Medical Association. Prior to sacrifice, animals were treated as described below for HRP transport analysis.

\section{Labelling Methods}

Anterograde Labelling of the Axons Within the Bridge (Intraocular Injection): Two days before sacrifice, animals used for intraocular injections were anesthetized with Nembutal as above. The left eyes were injected intraocularly with a $40 \%$ solution of HRP (Sigma type VI) in water. A Kopf microinjector was used to pressure inject $3 \mu \mathrm{L}$ of solution intraocularly over a period of 30 minutes. Any retinal ganglion cell axons projecting from the implant through the bridge should be labelled using this technique. A total of ten animals were prepared in this manner.

Retrograde Labelling of the Retina from the Bridge: Two days before sacrifice, additional animals that received a bridge were treated as follows. The skin over the bridge was excised over the skull region, and care was taken not to damage the bridge. The bridge appeared shiny white against the darker fascia, and was easily identifiable. With a No. 10 scalpel, the bridge was transected $5-6 \mathrm{~mm}$ from the burr hole. Gelfoam soaked HRP was applied directly to the transection site for 50 minutes. The Gel-foam was then removed and the proximal stump of the bridge was sealed with agar to prevent leakage of the HRP. The skin was then closed with wound clips over the bridge. A total of nine animals were prepared in this manner.

Retrograde Labelling of the Retina from the Forebrain: Two days before sacrifice, a third group of animals were treated as follows. The skin and a small piece of the skull over the implanted area of the forebrain were opened. One $\mu \mathrm{l}$ of a $40 \%$ solution of HRP was injected into the brain over 10 minutes, at a depth of $1-2 \mathrm{~mm}$. The skin was closed over the site and care was taken not to interfere with the bridge entering the brain. A total of five animals were prepared in this manner.

\section{Histology for HRP}

All histological procedures were performed in a blinded fashion, without prior knowledge of the treatment group. At the sacrifice time of 30 to 180 days post surgery, each animal was deeply anesthetized with sodium pentobarbitol and perfused transcardially with a solution of heparinized $0.9 \%$ saline followed by a fixative containing $1 \%$ paraformaldehyde, $2.5 \%$ gluteraldehyde and $4 \%$ sucrose in a $0.1 \mathrm{M}$ sodium phosphate buffer ( $\mathrm{pH} 7.3$ at $\left.4^{\circ} \mathrm{C}\right)$, followed by a $10 \%$ sucrose solution (at $4^{\circ} \mathrm{C}$ ). The eye, retina, proximal and distal bridge segment and brain were then removed and stored at $4^{\circ} \mathrm{C}$ in $10 \%$ phosphate buffered sucrose for 14 hours. The brain was then blocked and serial horizontal sections of the retina and bridge and coronal sections of the forebrain were cut at $50 \mu \mathrm{m}$ on a freezing microtome. Resulting sections were processed according to the tetramethylbenzidine (TMB) protocol of Mesulam /21/. Alternate sections were mounted on $2 \%$ gelatinized slides, air dried overnight and coverslipped the next day. A second set of slides were counterstained with thionin and coverslipped.

\section{RESULTS}

Removal of the embryonic eyes involved a number of technical problems that needed to be 
overcome. The first was that the eyes had to be maintained in vitro for as little time as possible. The more time that had elapsed from removal, suturing and implantation of the embryonic eyes, the poorer the axonal growth through the bridge. This was especially true of the E18 and E21 eyes.

The small size of the eyes (especially those of age E11) made suturing to the PNS segment difficult, even under a dissecting microscope. The suture needle was required to pass through the eye such that a portion of the retina as well as the sclera was impaled. A suture on either side of the eyeball to the nerve held the eye in position. A single knot of each suture was sufficient. The complex could then be gently manipulated with forceps.

It was also important to insure that the head of the embryonic optic nerve was abutted against the stump of the PNS nerve segment. This was most easily accomplished by passing the sutures on opposite sides of the embryonic globe, and securing it to opposite ends on the nerve segment.

Occasionally, the eye would roll and a third suture was required to re-orient the globe. Initially, we found it effective to secure the globe in vitro. This was accomplished by using a thin coat of paraffin on the bottom of the culture dish, and pinning the globe to the dish through any attached fascia with thin gauge needles. Eventually, proficiency with the preparation did not require this step to be performed.

Implantation of the embryonic eye/nerve complex into the host eye must be performed rapidly, as the preparation can become dry in air. Thus, the slit in the host eye should be performed first, and then the complex rapidly put into position. The end of the bridge should be trimmed so as to allow a minimum length from the eye to the burr hole (allowing an extra 3$4 \mathrm{~mm}$ for insertion into the burr hole). Finally, the eyelids should be sutured last. This is because the skin over the head may require more manipulation in the suturing process, which may dislodge the complex from the eye. Suturing the eyelids last will ensure that the complex is still intact by visual inspection.
No rats were lost due to post-surgical trauma. By the time of sacrifice, the eyelids of the host eyes became open, presumably because the animals scratched at the lesions and dislodged the sutures. However, the host eyes appeared healthy, and the implant was clearly visible through the cornea of the host eye as a white and red mass.

Histological results indicated that the implant developed into a distinctive structure (see companion paper). The implant was easily distinguished from the host as the implant developed in the vitreal environment close to (but not incorporated in) the host retina.

Retrograde labelling from the nerve bridge was shown to occur in the implanted (embryonic) retinal ganglion cells (see companion paper). The label was generally distributed in a lamellar pattern (Figure 5), and the layers of the retina could be distinguished in routine paraffin sectioning. In the forebrain the label was widely dispersed (Figure 6), and the HRP filled fibers could be seen to extend several millimeters throughout the forebrain in non-specific directions.

\section{DISCUSSION}

The technique described in this paper represents a novel approach to studying the growth potential of embryonic CNS axons in a clearly defined system. The technique combines other methodologies commonly used in CNS reconstruction, namely transplantation and CNS bridging with PNS grafts. This is the first attempt to combine these techniques in such a way as to enable transplanted embryonic CNS axons to redirect their growth into an adult host CNS via a PNS bridge.

In earlier studies, Aguayo et al /1/ sutured PNS bridges to the axotomized adult rat optic nerve. They found that the axons of the host retinal ganglion cells could regenerate through the bridge and enter the host brain. The axons were identified using HRP tracing techniques. In 
such cases where bridging neuronal structures with PNS segments occurs, the reactive Schwann cells of the bridge may afford the communicative means for regeneration to the target by providing diffusable growth-promoting factors $/ 27 /$, and by providing a suitable growth surface formed by the plasma membrane and/or basal lamina $/ 3 /$, or extracellular matrix /23/. However, even though CNS growth can occur over considerable distances in the PNS implant, the outgrowth is dramatically impeded when the axons approach the opposite CNS junction 19/. The reason why the PNS implant supports growth and the CNS does not is currently unknown. Theories concerning the differences between Schwann cells and glia with respect to their interactions with axons are receiving substantial attention (see /22/ for review).

The experiments of Aguayo and colleagues assessed the regenerative abilities of the adult CNS. In contrast, Blair and Turner /2/ studied the effect of developing systems in the adult CNS by grafting dissociated fetal or neonatal retinae into adult host retinae. The grafts were obtained from various age embryos or postnatal animals and were implanted into host retinae that had received a previous lesion (after various post-lesion times). The results indicated good survival of the grafts in virtually all combinations. This study not only emphasizes the influence of developing systems on those of adult structures, but demonstrates the ability of the host anterior chamber to provide necessary factors for development. Interestingly, in related studies Turner et al /26/ found that peripheral nerve segments implanted into the lesioned adult retina could initiate rescue of the host retinal tissue that would have otherwise died due to the lesion. The effect may have been related to a diffusable factor elaborated by the implant.

Del Cerro et al /6/ studied developing systems by transplanting retinae from 13-18 day embryos and 2 day postnatal rats into the anterior chamber of adult host rats of another strain. Light and electron microscopic analysis revealed that the transplants survived, differentiated and continued to develop to form what appeared to be ap- propriate layers of the retina. Therefore, the immunologically privileged anterior chamber supported the development of the embryonic cells, and enabled them to establish morphological characteristics reminiscent of normally developing retinae.

McLoon and McLoon /20/ have shown that reaggregated embryonic retinas placed over the superior colliculus of neonatal hosts will continue to differentiate and form projections to the target tissue. Even when the initial intercellular connections formed during early development are disrupted, these grafts are still capable of developing their characteristic laminae and synaptic connections /19/. Further, retinal transplants appear to project only to those targets which would normally receive retinal projections $/ 17 /$. When transplanted to adult superior colliculus, the dissociated retinae could still develop local projections, but did not appear as vigorous as when transplanted into newborns /18/. When fetal retina is transplanted to occipital cortex, the ganglion cells fail to survive beyond seven weeks /24/. In contrast, when the same age fetal retina was cotransplanted with tectum or diencephalon, the ganglion cells did survive up to 14 weeks $/ 24 /$. These studies suggest that appropriate target tissue may provide a trophic influence which is necessary for survival, maturation and axonal outgrowth.

The transplantation strategy has also been widely used in nonmammalian models in order to elucidate the processes of the formation of neuronal connections (for reviews, see $/ 4,12,16,25 /)$. Fundamental rules governing neurogenesis have been proposed using combinations of transplantation, electrophysiology and neuroanatomical tracing procedures (see /15/ for review). For example, experiments with transplanted Mauthner cells, olfactory placodes, eye primordia, and motoneuron targets indicate that there is a great deal of autonomy (use of intrinsic cues) in the initial outgrowth of axons of differentiating nerve cells; while the rules governing the long-range trajectory and morphology of these axons are different, involving extrinsic cues. Such cues may include gradients of diffus- 
able or substrate-bound materials released by the target. Other extrinsic cues do not appear to play a role in pattern formation such as mechanical guidance, fiber-fiber interactions, timing or the appropriate projection of pioneer axons.

In contrast to all of the above experiments, the present studies were designed to assess the redirected growth potential of a developing CNS structure through a PNS segment. This was accomplished by utilizing eyes of various age embryonic rats. The use of a bridge between the embryonic eyes and the host CNS allows the developing (and redirected) fibers to seek CNS target structures. Thus, by allowing the developing retinal system to establish such contacts, the host visual system can be partially "reconstructed". Towards this end, the companion paper to this report provides histological evidence of retinal development and projection into the host CNS. The question of functionality is the next primary issue to be addressed. As such, future use of this technique will ultimately establish whether synaptic connections can be formed, and whether such connections are functional.

\section{ACKNOWLEDGEMENTS}

This work was supported by grants from the New York State Science and Technology Foundation (MFZ) and from the Riland Neuromuscular Institute (BHH).

\section{REFERENCES}

1. Aguayo AJ, Vidal-Sanz M, Villegas-Perez MP, Bray GM. Growth and connectivity of axotomized retinal neurons in adult rats with optic nerves substituted by PNS grafts linking the eye and midbrain. Ann NY Acad Sci 1987; 495: 1-9.

2. Blair JR, Turner JE. Optimum conditions for successful transplantation of immature rat retina to the lesioned adult retina. Devel Brain Res 1987; 36: 257 270.

3. Bunge RP. Aspects of Schwann cell and fibroblast function relating to central nervous system regeneration. In Kao C, Bunge RP, Reier P (Eds), Spinal Cord Reconstruction. New York: Raven Press, 1983; pp 341-358.
4. Constantine-Paton M. Position and proximity in the development of maps and stripes. Trends Neurosci 1983; 6: 32-36.

5. Das GD. Neural transplantation in the spinal cord of adult rats. Conditions, survival, cytology and connectivity of the transplants. J Neurol Sci 1983; 62: 191210.

6. del Cerro M, Gash DM, Rao GN, Notter MF, Wiegand SJ, Sathi S, del Cerro C. Retinal transplants into the anterior chamber of the rat eye. Neuroscience 1987; 21: 707-723.

7. del Cerro M, Gash DM, Rao GN. Retinal transplants into normal and damaged adult retinas. Soc Neurosci Abstr 1987; 11: Part 1, 15.

8. del Cerro M, Gash DM, Rao GN, Notter MF, Wiegand SJ, Guipta M. Intraocular retinal transplants. Invest Ophthalmol 1985; 26: 1182-1185.

9. David S, Aguayo AJ. Axonal elongation into peripheral nervous system bridges after central nervous system injury in adult rats. Science 1981; 214: 106117.

10. David S, Aguayo AJ. Axonal regeneration after crush injury of rat central system fibers innervating peripheral nerve grafts. J Neurocytol 1985; 14: 1-12.

11. Forrester J, Peters A. Nerve fibers in optic nerve of rat. Nature 1967; 214: 245-247.

12. Fraser RS, Hunt RK. Retinotectal specificity: Models and experiments in search of a mapping function. Ann Rev Neurosci 1980; 3: 319-352.

13. Gan SK, harvey AR. Lack of ingrowth of retinal axons into the visually deafferented superior colliculus of young rats: A horseradish peroxidase study. Neurosci Lett 1986; 70: 10-16.

14. Greene HSN. The use of transplanted tissues in biology and histology. In: Bourne GH (Ed), In Vivo Techniques in Histology. Baltimore: Williams and Wilkins, 1967; pp 80-112.

15. Harris WA. Neural transplants in lower vertebrates. In Sladek JR, Gash DM (Eds), Neural Transplants: Development and Function. New York: Plenum, 1984; pp 43-98.

16. Horder TJ, Martin KAC. Morphogenesis as an alternative to chemospecificity in the formation of nerve connections. Symp Soc Exp Biol 1978; 32: 275-358.

17. McLoon SC, Lund RD. Specific projections of retina transplanted into rat brain. Exp Brain Res 1980; 40: 273-282.

18. McLoon SC, Lund RD. Development of fetal retina, tectum, and cortex transplanted to the superior colliculus of adult rats. J Comp Neurol 1983; 217: 376389.

19. McLoon SC, Lund RD, McLoon LK. Transplantation of reaggregates of embryonic neural retina to neonatal rat brain: Differentiation and formation of connections. J Comp Neurol 1982; 205: 179-189.

20. McLoon SC, McLoon LK. Transplantation of the developing mammalian visual system. In Sladek JR, Gash DM (Eds), Neural Transplants: Development 
and Function. New York: Plenum, 1984; pp 99-124.

21. Mesulam M-M. Tetramethylbenzidine for horseradish peroxidase neurohistochemistry: A noncarcinogenic blue reaction product with superior sensitivity for visualizing neural afferents and efferents. J Histochem Cytochem 1978: 26: 106-117.

22. Reier PJ. Neural tissue grafts and repair of the injured spinal cord. Neuropath Appl Neurobiol 1985; 11: 81-104.

23. Sanes JR, Marshall LM, McMahan UJ. Reinnervation of muscle fiber basal lamina after removal of myofibers. J Cell Biol 1978; 78: 176-198.

24. Sefton AJ, Lund RD, Perry VH. Target regions en- hance the outgrowth of ganglion cells in embryonic retina transplanted to cerebral cortex in neonatal rats. Dev Brain Res 1987; 33: 45-149.

25. Sladek JR, Gash DM (Eds), Neural Transplants: Development and Function. New York: Plenum, 1984, $454 \mathrm{pp}$.

26. Turner JE, Blair JR, Chappel ET. Peripheral nerve implants effects on survival of retinal ganglion layer cells after axotomy initiated by a penetrating lesion. Brain Res 1987; 419: 46-54.

27. Varon S, Manthorpe M, Williams LR. Neuronotrophic and neurite promoting factors and their clinical potential. Dev Neurosci 1983; 6: 73-100. 

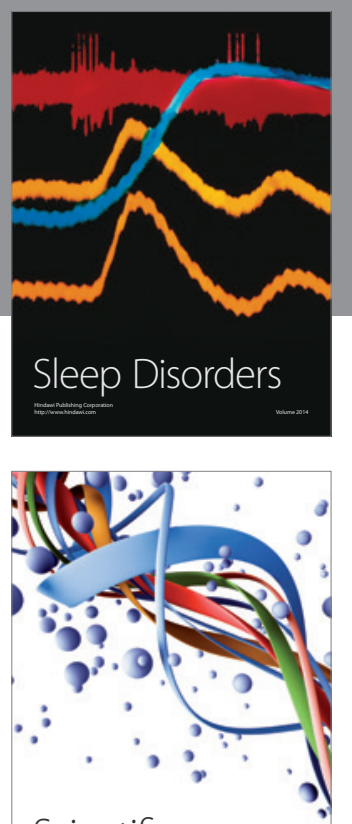

Scientifica
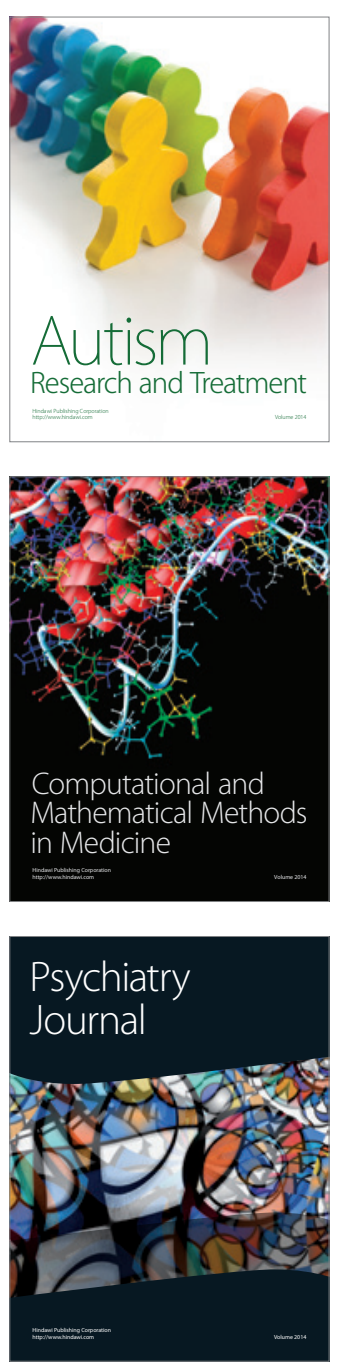
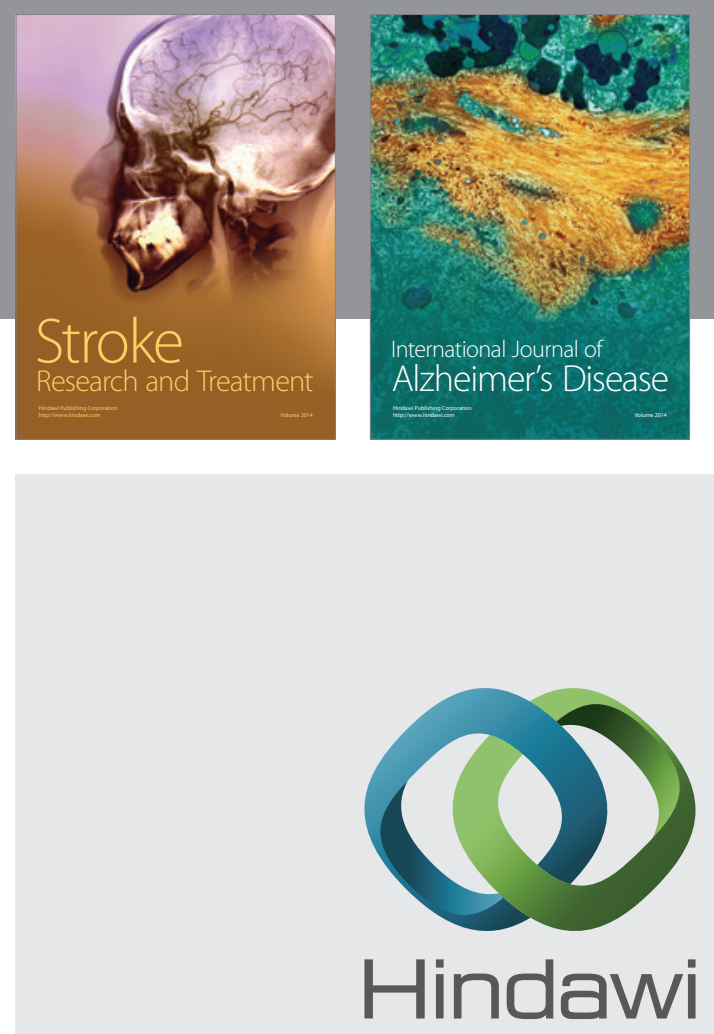

Submit your manuscripts at

http://www.hindawi.com
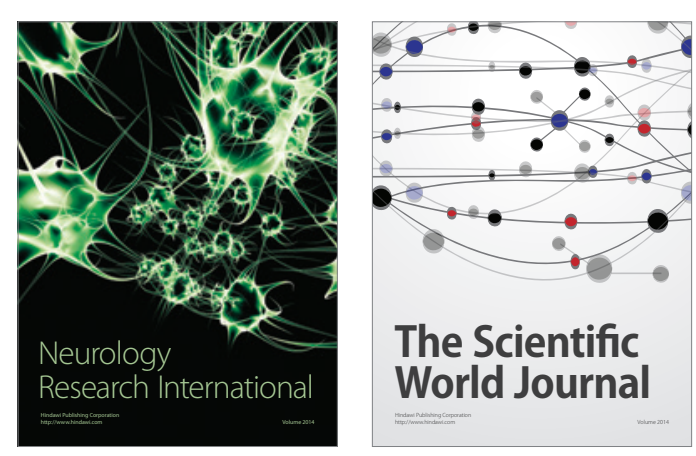

The Scientific World Journal

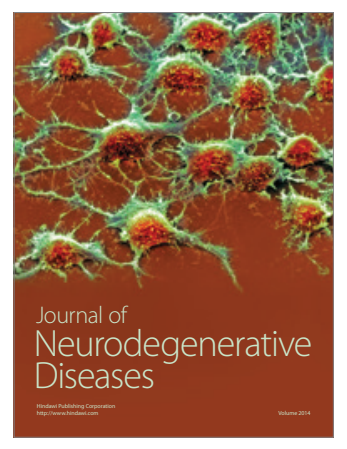

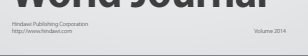

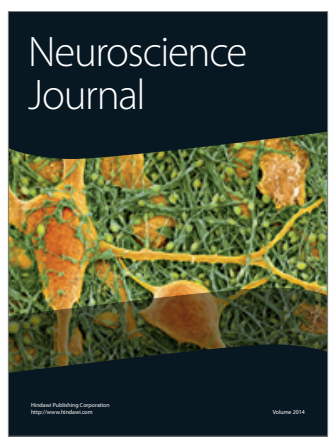

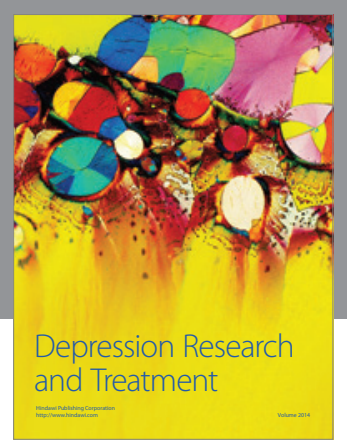
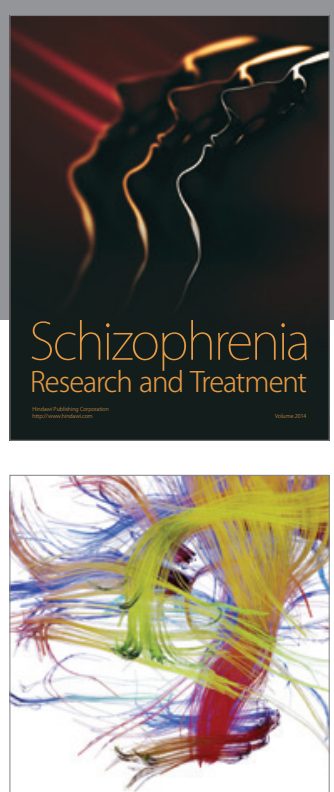

Brain Science

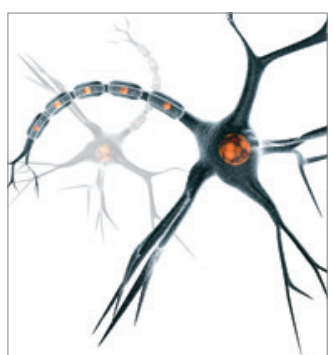

Neural Plasticity
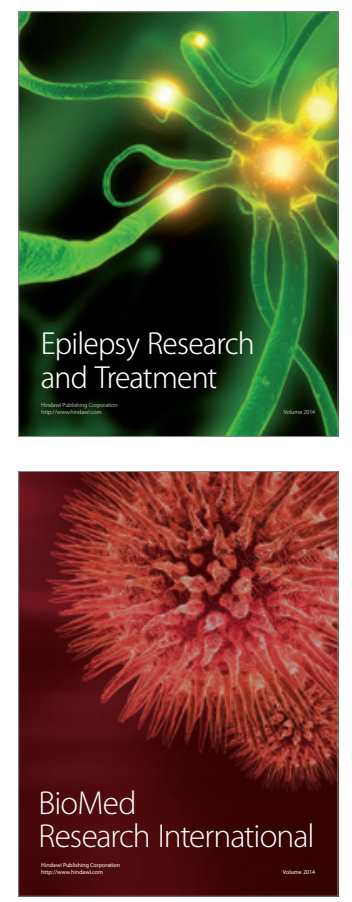

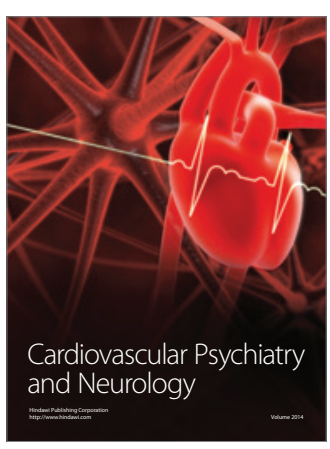

Parkinson's

Disease
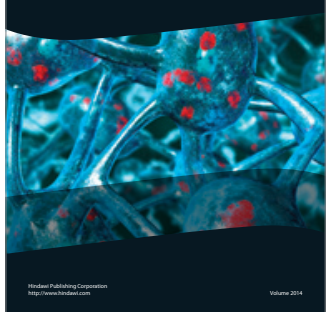